\title{
Application of Traditional Cultural Symbols in Art Design under the Background of Artificial Intelligence
}

\author{
Cuifang Lin \\ College of Animation and Digit Art, Anhui Sanlian University, Hefei 230601, Anhui, China \\ Correspondence should be addressed to Cuifang Lin; 20200120697@nxmu.edu.cn
}

Received 19 July 2021; Revised 13 September 2021; Accepted 17 September 2021; Published 26 October 2021

Academic Editor: Sang-Bing Tsai

Copyright (C) 2021 Cuifang Lin. This is an open access article distributed under the Creative Commons Attribution License, which permits unrestricted use, distribution, and reproduction in any medium, provided the original work is properly cited.

\begin{abstract}
In order to solve the declining influence of traditional cultural symbols, the research on traditional cultural symbols has become more meaningful. This article aims to study the application of traditional cultural symbols in art design under the background of artificial intelligence. In this paper, a fractal model with self-combined nonlinear function changes is constructed. By combining nonlinear transformations and multiparameter adjustments, various types of fractal models can be automatically rendered. The convolutional neural network algorithm is used to extract the characteristics of the style picture, and it is compared with the trained picture many times to avoid the problem of excessive tendency of the image with improper weight. The improved L-BFGS algorithm is also used to optimize the loss of the traditional L-BFGS, which improves the quality of the generated pictures and reduces the noise of the chessboard. The experimental results in this paper show that the improved L-BFGS algorithm has the least loss and the shortest time in the time used for more than $500 \mathrm{~s}$. Compared with the traditional AdaGrad method, its loss is reduced by about $62 \%$; compared with the traditional AdaDelta method, its loss is reduced by $46 \%$. Its loss is reduced by about $8 \%$ compared with the newly optimized Adam method, which is a great improvement.
\end{abstract}

\section{Introduction}

At present, artificial intelligence has reached a very popular level. Many giant companies at home and abroad will not hesitate to spend a lot of money to recruit talents to study various fields of artificial intelligence technology. This means that artificial intelligence will occupy a very important position in the market. From the perspective of big data, China's huge Internet population can be called a big Internet country, which can generate massive amounts of data every day, which provides more sufficient data for the learning of artificial intelligence algorithms than other countries. Artificial intelligence is currently being explored to varying degrees in different fields, and the field of the art design is an important base for artificial intelligence technology to be practiced, especially in interactive art design. In the development history of human civilization for thousands of years, the transformation of production tools and production methods has often played an epoch-making significance. The transformation of early humans was an evolutionary process from ape-man to Homo sapiens. In fact, it can also be seen as a process of major changes in production tools. That is to say, the continuous development of intelligent technology will bring about changes in productivity in different fields. Innovative research that causes breakthroughs, of course, also includes the field of interactive art design. Nowadays, more attention should be paid to a humanized interactive experience. The combination of artificial intelligence and interactive art design is an exploration of current hot topics. The application of artificial intelligence in interactive art design not only can bring changes to the form of interactive art design but also can identify whether it will be replaced by machines. The unknowingness of this poses a psychological threat to human beings. The traditional art field is receiving the impact of the emerging industry of artificial intelligence. If traditional art and artificial intelligence are not combined, traditional art and traditional cultural symbols will slowly decline.

With the rapid development of computer technology and the Internet, the interactive nature of art design has 
become more prominent and distinct. The existence of a computer as a control device manages the process of interaction in an unprecedented way. Based on the current intelligent technology involved in interactive art design, artificial intelligence has also begun to gradually appear in daily life. The intelligent interactive art design can respond to the recipient's behavior, posture, voice, expression, temperature, remote control, and climate and environmental changes through smart robots, computers, sensors, smart programs, smart materials, and other smart tools. The combination of the interactive art design and artificial intelligence is an extension of traditional creative forms. With the changes brought about by the intervention of smart technology, it also brings new problems, but at the same time, it also gives a new perspective to look at art. Through the research of this article, it is hoped that more scholars will pay attention to the content of artificial intelligence and interactive art design theory so that intelligent interactive art design can make better progress in the current intelligent technology environment and provide for artificial intelligence and interactive art design [1].

Under the current artificial intelligence background, the importance of traditional cultural symbols is slowly decreasing. The following people have their own opinions on this. Song pointed out that the Internet of Things is rapidly becoming a business opportunity and home interior design technology and standards, mainly wireless communication between the equipment and tools of human daily life, generally called the Internet of Things. The artistic design of the product is behind the home furnishings, as well as the service for the electrical appliances connected to the entire home control system Internet. This service allows users to decorate their home's interior design while building an IoT and field programmable gate array environment. Smart home systems have gained popularity in the past few decades because they have improved comfort and quality of life. Smartphones and microcontrollers use wireless communication technology to control and integrate the features of smart home systems that control room furniture and the Internet of Things to control smartphone applications, sensor triggers, and smart Internet support. Cloud computing technology is embedded in the content of the smart home. Although he proposed the combination of the Internet of Things and art design, the scope of his combination is too narrow. He believes that the scope is currently limited to furniture, which does not give full play to the use of the Internet of Things [2]. Jia proposes more and more diversified environmental art design, combined with advanced virtual reality technology, and has made a huge contribution to the development of modern display design. There is a screen in front of you, and the sound can be transmitted through speakers, which make people have an exciting feeling. The application of virtual reality technology in environmental art must follow three principles: the principle of authentic content, the principle of a formal application, and the principle of the quantitative review. Although he wants to diversify environmental art and combine it with virtual reality, the limitations of virtual reality itself are not used in environmental art. After all, artificial simulation cannot be $100 \%$ similar [3]. Zhu proposed that with the continuous development of artificial intelligence, the application of artificial intelligence will no longer repeat engineering disciplines such as "science and technology," "medical research and development," and "computer." In recent years, artificial intelligence has also been fully developed and innovated in the field of "art." Among them, 3DPT has changed the traditional manufacturing mode and is completely different from the traditional one. Therefore, it is necessary to be precise in the shape and structure of the product without increasing additional costs. With 3DPT, complex processes can be better completed, which will greatly reduce time costs. Although he proposed the impact of $3 \mathrm{D}$ printing technology on the traditional art industry with the help of artificial intelligence, this is not a long-term impact. The traditional art industry can also be developed in combination with 3D printing, which can completely turn its disadvantages into the driving force for its own development [4].

The main innovations of this paper are: (1) constructing a fractal model with self-combined nonlinear function changes. By combining nonlinear transformations and multiparameter adjustments, multiple types of fractal models can be automatically rendered. (2) The convolutional neural network algorithm is used to extract the characteristics of the style picture, and it is compared with the trained picture many times to avoid the problem of excessive tendency of the image with improper weight. (3) The improved L-BFGS algorithm is used to optimize the loss of the traditional L-BFGS, which improves the quality of the generated pictures and reduces the noise of the chessboard.

\section{Methods on the Application of Traditional Cultural Symbols in Art Design under the Background of Artificial Intelligence}

2.1. Background of Artificial Intelligence. When many people hear about artificial intelligence, they associate it with robots, but the concepts of the two are not the same. "Intelligence" can also be regarded as "wisdom" and "ability," the combination of the two has the meaning of intelligence [5]. Although both human intelligence and artificial intelligence contain "intelligence," they cannot be equated. What distinguishes humans from artificial intelligence is that humans have a dreaming subconscious. Humans dreamed of creating some tools or machines to solve all kinds of manual labor. Just like the emergence of the steam engine in the eighteenth century, the arrival of the information revolution in the 1940s, and the emergence of artificial intelligence in the 1950 s, these are the results of human dreams and put them into practice [6]. Therefore, it can be understood that artificial intelligence is created and developed by humans, is an incomplete simulation and extension of human intelligence, and is also a component of human intelligence [7].

As a field of human intelligence research, artificial intelligence has gone through years and generations of efforts from the initial mechanical automation to electronic 
automation to the current information automation [8]. However, the current artificial intelligence is still in the stage of weak artificial intelligence. In fact, the most fundamental difference between strong and weak artificial intelligence is the processing of logic formation. Strong artificial intelligence completes a series of learning activities by itself as the main body, and it is not affected by the active acquisition. Human constraints can develop independently, while weak artificial intelligence is passively accepted and passively perform tasks according to the requirements of the designed program. Daniel Dennett believes that a bottom-up approach such as evolutionary algorithms can make artificial intelligence close to human intelligence, but he believes that the emergence of such strong artificial intelligence will bring about many ethical issues. In short, both weak and strong artificial intelligence are manifestations of human intelligent behavior design, and their research and development can be used to replace human labor $[9,10]$. Weak artificial intelligence has safety and reliability, while strong artificial intelligence has higher work efficiency. In the era of rapid technological development, although the two are controversial, they actually jointly promote the development of artificial intelligence.

\subsection{Cultural Symbols. Generally speaking, each cultural} system has its own symbol system, and symbol is the medium to realize the communication of people in the organization. If communication cannot be carried out and meaning cannot be conveyed, then the culture that means "holistic way of thinking and living habits" will no longer exist [11]. At the very beginning, people did not realize that they belonged to a certain culture until they encountered "cultural differences." This "cultural difference" is not only the difference in form of expression from other cultures, but the deeper level is the interpretation of symbols or the differences caused by the different "meanings" given to the symbols by the cultural system [12]. First, it is to be understood from the "unconsciousness" of language structure. The main content of the Sapir-Wolf hypothesis is simply: what kind of world people face is determined by what kind of language they use. Moreover, the "clue" can be seen from the language used. It cannot be denied that the Sapir-Wolf hypothesis has its limitations: it is almost onesided "linguistic determinism." This kind of misunderstanding of thinking may fall into a static worldview system, and it may also make people lose confidence in crossborder. In fact, one of the inspirations of the Sapir-Wolf hypothesis is that when exploring a culture, one must find the "stick that moves the Earth," and to find this "stick" is to explore the "language of speech," in other words, the beginning of exploring the meaning given to a certain symbol by the members of the cultural organization [13]. It can be said that this is the reason why "symbols" are chosen as the entry point for the study of traditional cultural dissemination [14]. Eco is known as one of the "four great masters of systematic semiotics" (the other three are: Saussure, Peirce, and Greimas). Unexpectedly, his views have been recognized by many people, which have also led some scholars to an extreme: the emergence of "overinterpretation" [15]. In order to rebuild the objectivity foundation of the text, Eco has thrown out a new concept: "the intention of the text" and use it as the standard for interpreting the text [16]. He believes that "the intention of the text" plays an important role in the process of generating the meaning of the text. In this way, when interpreting symbols, it not only can satisfy the openness and generation of the text but also determine certain limits and standards for understanding and interpretation; this is also the goal that the author hopes to achieve when analyzing symbols. Eco also believes that the study of culture should be based on the signification system. Simply put, if you can use semiotics to analyze some self-evident cultural phenomena, you can get a clearer explanation and find its potential formation rules or mechanisms [17]. Through the above analysis, it is concluded that members outside cultural organizations recognize different cultures through cultural symbols, that is, to understand the culture from the representation of cultural symbols; members within cultural organizations realize cultural identity through cultural symbols, that is, construct from the connotation of cultural symbols culture.

2.3. Fractal Art. People are tired of the busy daily office in life, so spiritual comfort is particularly important. One of the functions of art can have the effect of alleviating people's mental stress [18]. However, in traditional concepts, not everyone can do art creation. Duchamp's urinal pulled the artist off the altar and opened the era of "everyone is an artist." The emergence of smart terminal devices based on mobile Internet and microelectronics technology seems to have made everyone engaged overnight. In such an era of big data, the huge amount of digital virtual art flooding the virtual space of the network, as well as various interactive and instant communication platforms developed to facilitate communication, provide a great convenience for the realization of art simulation. Such a large environment also provides a practical technical condition for producing fractals of complex and gorgeous pictures [19]. A large number of computer art and virtual art are flooded on the Internet. Such a large environment and platform provide convenience for the realization of art simulation and provide a feasible theoretical technical means that is easier to generate fractals of complex and gorgeous pictures. As mentioned earlier, the fractal is a branch of nonlinear science, and it has been widely used in various fields. Artworks are created based on different theoretical methods, and it has more advantages based on fractal theory. First, due to the intervention of fractal theory, the advantages will be manifested. There are countless possibilities of fractal characteristics. Only by changing the value of the parameter can form the fractal pattern and the ever-changing fractal three-dimensional modeling. Second, because fractals are generated by iterative algorithms; only the information needs to be stored in the transformation process, and there is no need to store a large amount of pixel information, which provides a 
platform for online creation, submission, and interaction in terms of practical possibilities. The fractal theory derives its own self-similar properties and creates many works, which make its structure have infinitely varying fineness. Based on fractal theory, images not only can produce fine branches but also can achieve faster data transmission. Instant online interaction allows creators to experience the charm of art. Third, borrowing the editing commands of professional graphics processing software can produce pictures with higher artistic aesthetics. To make the graphics produce stronger artistic effects, it is necessary to process the designed fractals through commands such as copy, reproduction, superimposition, and rotation sequence and can also convert the structure of three-dimensional modeling so that it has a higher level of artistic value. Fractal works of art can allow creators to be more immersed and experience in the realm of art. Therefore, that fractal art can be active on the stage of contemporary art [20].

People will have new ideas to study the morphology and structure of objects and will try to describe nature or physical phenomena from another perspective. Mainly due to the birth of fractal geometry, it provides an indispensable theoretical foundation for the birth of fractal art [21].

2.4. Fractal Geometry Aesthetics. The graphics based on fractal theory have attracted people's attention from their appearance to their development today. They have a wide range of applications, and their visual effects are shocking. With the perfect combination of craftsmanship and art, its practical value is even more important and paves the way for future fractal research. With the rapid development of contemporary science and technology, art has also entered the field of computer production such as science fiction movie scenes, architectural design, interior design, conceptual clothing design, and so on. There will be more and more fractal daily necessities and daily necessities in life. All necessities catch the attention of the public [22, 23].

Discovering the problem is one of the motives of artistic creation, and the existence of the problem is also the artistic creation. In the beginning, artistic creation was a connection between one's own thoughts and technology. Artworks cannot be copied and cannot be recreated. This is the only work of art, which is the understanding of artworks in the traditional sense [24]. The level of artistic creativity is also judged by it. As an innovative work of art, it can be grasped from the theoretical direction and can also be discussed from the perspective of science and art. Individual creators use their skills and language freely in order to connect and influence contemporary art with the reconstruction of individual disciplinary systems. Artworks are composed of different disciplines, and globalization is ultimately formed by individuals. It is a historical development trend and an inevitability of social development. The inevitable connection between disciplines made me ask for the cause from multiple disciplines [25].

As shown in Figure 1, linear and nonlinear fractals describe the difference in the content of fractal geometry. Graphs have self-similarity, irregular arrangement, and continuous dimensionality transformation. Generally, fractal objects that can be described by one fractal dimension belong to linear fractal graphics. The graphics processed by nonuniform linear transformation or nonlinear transformation are nonlinear fractal graphics. Nonlinear fractals can be divided into three types: self-affine fractals, self-inversion fractals, and self-square fractals.

As shown in Figure 2, it is a linear image display, in which nonlinear images are superior to their own characteristics and can be spliced together. Linear images can only be spliced under certain conditions. It is almost impossible for Euclidean geometry to be produced visually by breaking through the sense of edges and corners and being broken by fractal geometry. Most objects that can be seen by the human eye can be represented by Euclidean geometry, such as desks, ships, beds, built buildings, and so on, which are composed of two- and three-dimensional objects. However, some scenes that usually exist in nature, such as the starry sky and cumulus clouds, cannot be represented by well-known geometric figures. Therefore, the birth of fractal geometry brings different description angles to some natural scenes. Fractal geometry describes these subtle differences and the mapped objects through the idea of fractal dimensions and the use of different dimensions.

2.5. Self-Assembling Nonlinear Transformation Modeling. The traditional two-dimensional function iteration follows the linear transformation model and only uses an affine transformation to complete the nesting of self-similarity [26]. The affine transformation uses a $3 \times 3$ matrix to describe the translation, scaling, flipping, rotation, and shearing of the image. The transformation matrix is as follows:

$$
\left[\begin{array}{c}
y^{\prime} \\
x^{\prime} \\
1
\end{array}\right]=\left[\begin{array}{ccc}
n_{00} & n_{01} & n_{02} \\
n_{10} & n_{11} & n_{12} \\
0 & 0 & 1
\end{array}\right]\left[\begin{array}{l}
y \\
x \\
1
\end{array}\right]=\left[\begin{array}{c}
n_{00} * y+n_{01} * x+n_{02} \\
n_{10} * y+n_{11} * x+n_{12} \\
1
\end{array}\right] \text {. }
$$

The following operations has to be performed on the matrix:

Translate

$$
\left[\begin{array}{llc}
1 & 0 & t y \\
0 & 1 & t x \\
0 & 0 & 1
\end{array}\right]
$$

Spin

$$
\left[\begin{array}{ccc}
\cos \alpha & -\sin \alpha & 0 \\
\sin \alpha & \cos \alpha & 0 \\
0 & 0 & 1
\end{array}\right]
$$

Zoom

$$
\left[\begin{array}{ccc}
s y & 0 & 0 \\
0 & s x & 0 \\
0 & 0 & 1
\end{array}\right]
$$




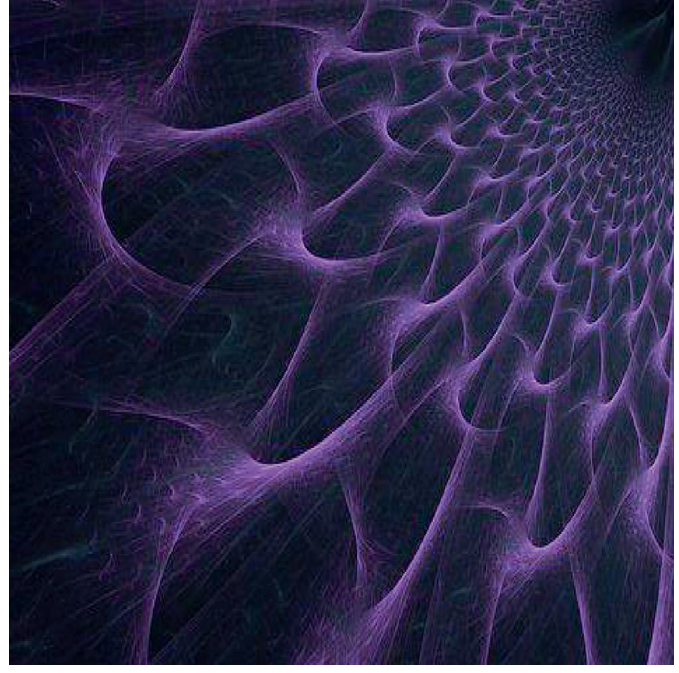

Non-linear fractal picture

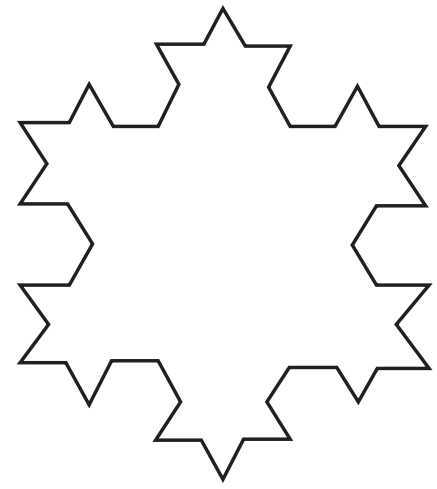

Linear fractal picture

FIGURE 1: Linear and nonlinear graphics.

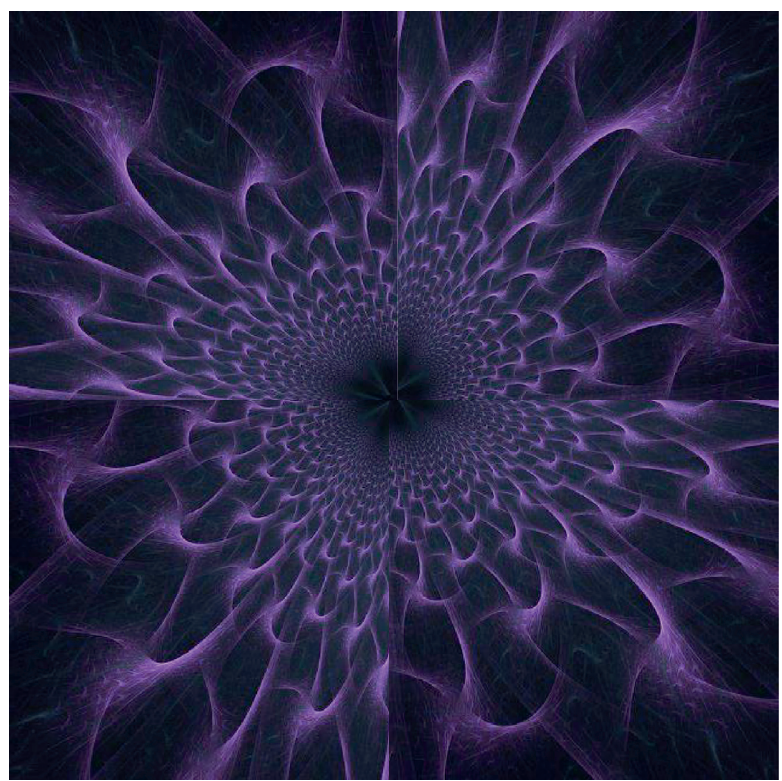

Non-linear fractal picture display

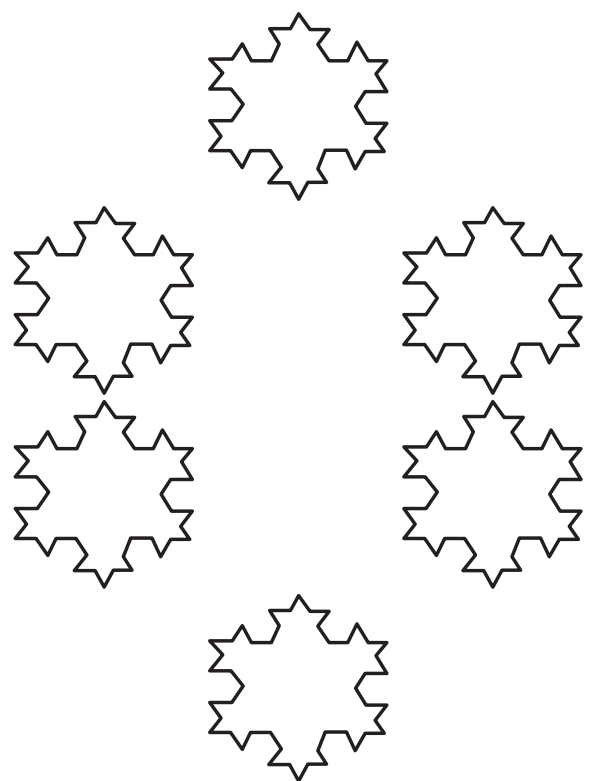

Linear fractal picture display

FIgURE 2: Linear fractal image display.

Cut

$$
\left[\begin{array}{ccc}
1 & \tan \gamma & 0 \\
\tan \lambda & 1 & 0 \\
0 & 0 & 1
\end{array}\right]
$$

The shear transformation matrix is equivalent to the composite of horizontal and vertical shear as follows:

$$
\left[\begin{array}{ccc}
1 & 0 & 0 \\
\tan \lambda & 1 & 0 \\
0 & 0 & 1
\end{array}\right]\left[\begin{array}{ccc}
1 & \tan \gamma & 0 \\
0 & 1 & 0 \\
0 & 0 & 1
\end{array}\right] .
$$

Traditional linear affine transformation is based on different affine matrices to achieve the effect of affine transformation as follows:

$$
F_{j}(y, x)=\left(b_{j} y+a_{j} x+d_{j}, c_{j} y+f_{j} x+e_{j}\right) .
$$

The nonlinear transformation $V_{i}$ is added to the general affine transformation to obtain

$$
F_{j}(y, x)=V_{i}\left(b_{j} y+a_{j} x+d_{j}, c_{j} y+f_{j} x+e_{j}\right) .
$$

Each nonlinear variable $V_{i}$ changes identifiable features such as the shape of the graph. By assigning different weight 
coefficients $v_{i j}$ to the nonlinear attraction factor $V_{i}$, the transformation effect is further expanded as follows:

$$
F_{j}(y, x)=\sum_{i} v_{i j} V_{i}\left(b_{j} y+a_{j} x+d_{j}, c_{j} y+f_{j} x+e_{j}\right) .
$$

First, an affine transformation is performed on a set of coordinates, and then the nonlinear transformation is applied to the result of the affine transformation. For the result of nonlinear transformation, a post-transformation that can control the coordinates is provided. It can be seen that the function $q_{j}(y, x)$ is derived from the second-order affine transformation of the nonlinear result

$$
q_{j}(y, x)=\left(\gamma_{j} y+\lambda_{j} x+\eta_{j}, \sigma_{j} y+\theta_{j} x+\tau_{j}\right) .
$$

Based on the above improvements, the function iteration process formula is as follows:

$$
F_{j}(y, x)=q_{j}\left(\sum_{i} v_{i j} V_{i}\left(b_{j} y+a_{j} x+d_{j}, c_{j} y+f_{j} x+e_{j}\right)\right) .
$$

Figure 3 shows a single iterative primitive. First, the data is subjected to the first affine transformation, followed by the nonlinear transformation, and then the second affine transformation, also known as post-transformation. For the nonlinear transformation, the combined nonlinear function can be designed to process the affine result, and the nonlinear transformation coefficient $v_{i j}$ can be controlled to adjust the pattern.

$$
\begin{aligned}
\sigma & =\sqrt{x^{2}+y^{2}} \\
\theta & =\arctan \left(\frac{x}{y}\right) \\
V_{1}(x, y) & =\frac{\cos \theta+\sin \theta}{\left(a_{1} * \sigma^{3}+a_{2} * \sigma^{2}+a_{3} * \sigma\right)}(x, y), \\
V_{2}(x, y) & =\left(e * \frac{\ln (x)}{1+x^{2}}, y\right) \\
V_{3}(x, y) & =\frac{(x \cos \theta+y \sin \theta)(x, y)}{\sigma} .
\end{aligned}
$$

After determining the structure, you can make different combinations based on the transformation function to write a new two-dimensional image transformation function, where $\sigma$ and $\theta$ in the transformation function are shown above.

\section{Experiment on the Application of Traditional Cultural Symbols in Art Design under the Background of Artificial Intelligence}

Due to the design differences of fractal graphics, some of the rendered graphics patterns will be concentrated, and the background difference between the front and back is obvious. Because the design is usually used for fabric printing and dyeing, the local style migration causes the edge pixels to be blurred, and the front and back styles are very different. The local style migration is not suitable for use. In view of the specificity of the fractal Atlas, through the analysis of image iteration, model iteration, and different loss functions, the style transfer algorithm based on perceptual loss is compared and optimized. According to the brightness and texture difference of the style picture, a reasonable initial style weight is set to ensure the image rendered by the trained generative model adds the style texture feature of the style image on the basis of keeping the content intact.

3.1. Experimental Subjects. Different fractal algorithms are used to generate graphics iteratively, and the fractal graphics lines are rendered to obtain the final graphics. The traditional cultural symbols are selected as the experimental objects, the selection criteria are symbolic pictures, and the exclusion criteria are pictures that are fuzzy and not clearly positioned.

3.2. Experimental Steps. Iteratively generate graphics from traditional symbols, put the resulting pictures into a convolutional neural network for training, optimize the resulting pictures, and finally adjust the parameters to obtain the final image.

3.3. Experimental Method. The single iterative primitive method is used for affine changes and nonlinear changes, and in actual demand, the combination of self-designed and secondary affine transformations are used. The convolutional neural network is used to train the image for multiple iterations, and the appropriate image is selected. Then the L-BFGS loss optimization method is used to improve image quality and reduce noise. The image features extracted through training are compared multiple times to avoid the problem of excessively tilted images with improper weights, summarize the appropriate style weight interval, and finally adjust the parameters to automatically render multiple types of fractal images, breaking the single artistic style, realizing the secondary development of fractal graphic art.

\section{Application of Traditional Cultural Symbols in Art Design under the Background of Artificial Intelligence}

4.1. Graphical Iterative Analysis. One of the differences between fractal geometry and traditional Euclidean geometry is that the dimensions of traditional Euclidean geometry are all positive integer dimensions, but the dimensions of fractal graphics are allowed to exist fractal dimensions, and most of the dimensions, fractal graphics.

As shown in Figure 4, the unit line segment is first divided into three equal parts, and then the middle part is replaced with two polylines, and then each line segment of the new line segment is divided into three equal parts, and 


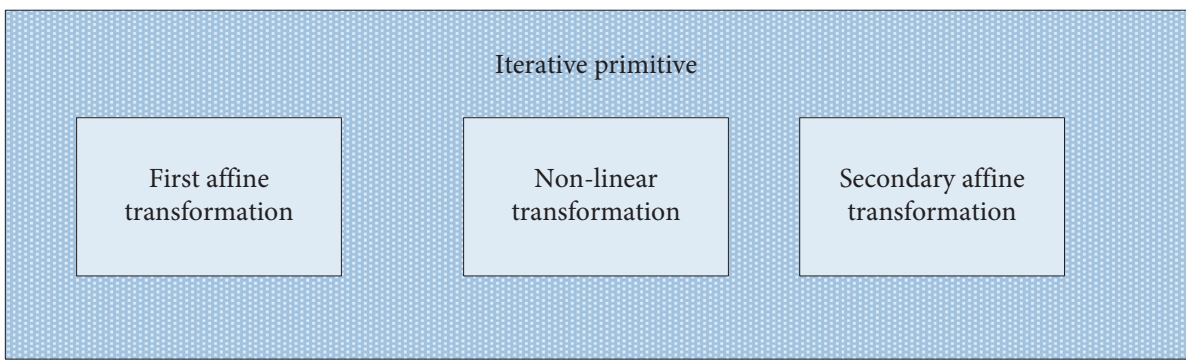

FIGURE 3: Single iteration primitive.

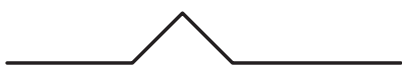

(b)

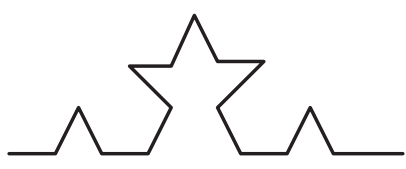

(c)

FIGURE 4: Construction steps of the fractal curve.

the middle part is replaced by half and half of the polylines. Repeating this iteration, after infinite iterations, a new fractal curve is generated.

As shown in Figure 5, this is the graph obtained after countless iterations. It is also the famous Koch curve. This curve has the characteristics of strong local correlation, simple structure, and strong local similarity.

Figure 6 is the Koch curve obtained after the first affine. The first affine transformation adopts the characteristic of self-similarity of the transferable figure and then takes advantage of the self-similarity between the local and the whole of the geometric object.

Figure 7 is derived from the first affine transformation of the Koch curve and then the nonlinear transformation. With this change, users can also modify the parameters according to their own needs to obtain the image they want.

Figure 8 is obtained by combining the Koch function through different transformation functions. It is formed by different permutations and combinations of its initial coordinates, plane rotation coefficient, interlayer weight coefficient, and the number of iterations within the range of values. By setting different parameters, the final image will also be different. Put the final image data into the convolutional neural network for training, you can filter to get more robust pictures.

4.2. Graphic Texture Feature Extraction and Analysis. As shown in Figure 9, each graphic has different content weights and different style weight ratios. These factors will cause pictures to have different biases. When the style weight ratio exceeds the content weight ratio beyond a certain range, the graphic structure of the content image will be damaged. Using digital image processing technology, the computer automatically extracts the pattern of the picture and organizes it to generate a continuous pattern of squares, which can assist the designer in creating more quickly and improve the efficiency of creation.

Four-side pixel extraction is performed on the edges of the fractal image and then converted into a grayscale image. Subsequently, the texture feature is extracted through the gray level co-occurrence matrix, the texture gray similarity is calculated, and the $H$ value is calculated to determine the uniformity of the gray texture.

As shown in Table 1 , the $H$ values of serial numbers a and $b$ are both greater than 2 , indicating that the foursided texture is simple and uniform and suitable for image splicing operations. The pictures suitable for splicing is filtered out, and then the next splicing operation is performed.

As shown in Figure 10, they are scattered splicing and continuous splicing. Aiming at the characteristics of selfaffine, infinite fineness, and independent pattern of fractal graphics, this kind of graphics can be spliced by scattered points in serial number a in Table 1, which requires distinct pattern characteristics, uniform distribution, and regular distribution, and can also be scattered freely. The continuous splicing of Figure performance.

As shown in Figure 11, in the time it takes for different optimization functions to iterate 1,000 times and the impact on the loss function, the gradient of the entire image has relatively large fluctuations, and the convergence speed is slow. A more optimal optimization method can be selected. Among them, the time used by the L-BFGS algorithm is the 


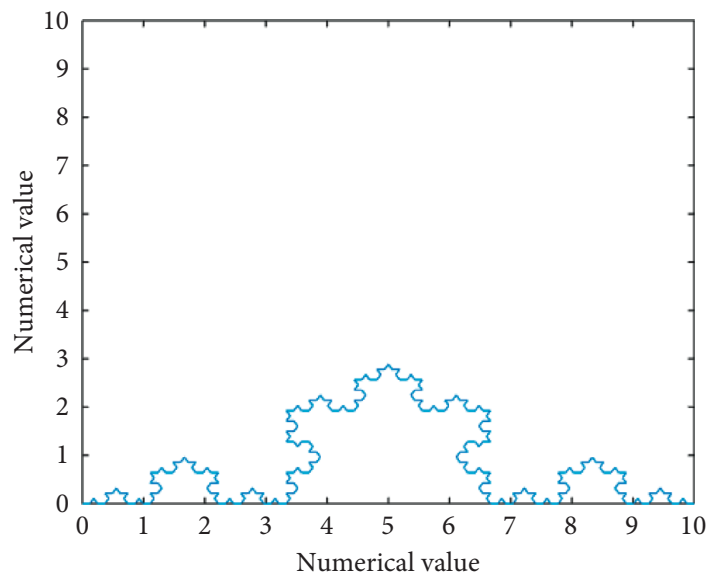

FIGURE 5: Curve after iteration.

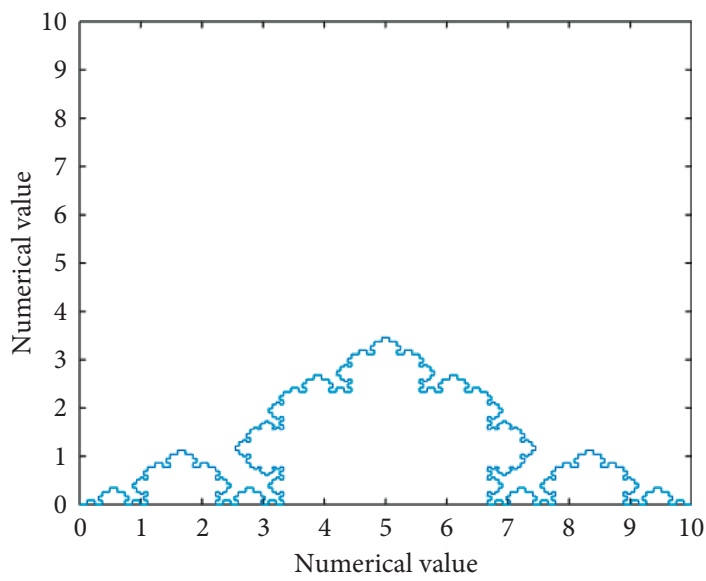

Figure 6: The first affine.

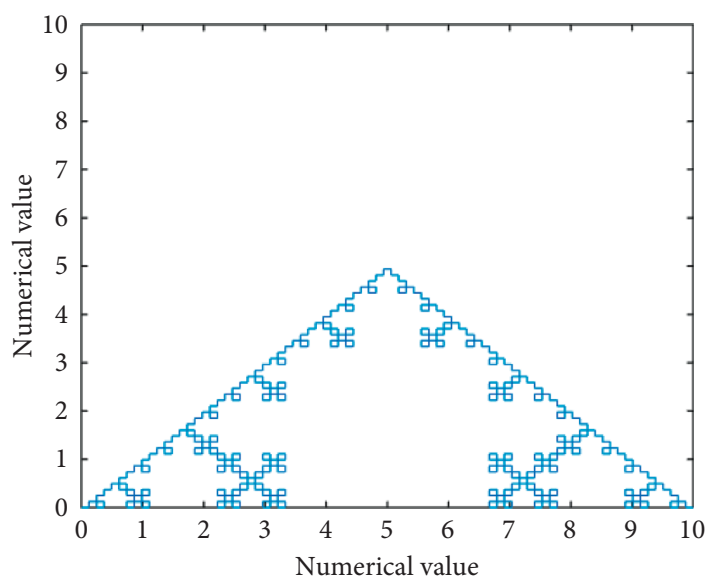

FIGURE 7: Nonlinear transformation.

shortest, and the loss is the smallest. In the time used for $500 \mathrm{~s}$ and above, its loss is reduced by about $62 \%$ compared to the traditional AdaGrad method, and $46 \%$ less than the traditional AdaDelta method. The loss is about $8 \%$ less than the newly optimized Adam method. Compared with traditional artificial intelligence algorithms, the reduction of these damages is very effective and obvious for the improvement of algorithm performance. 


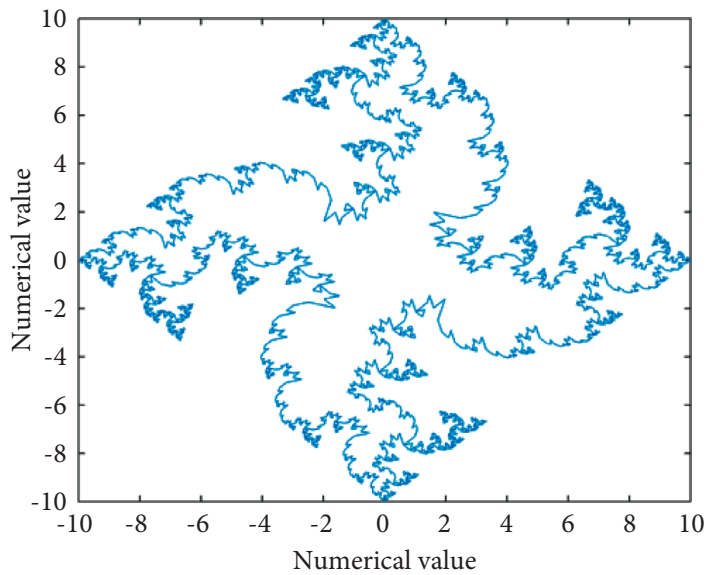

Figure 8: The second affine.

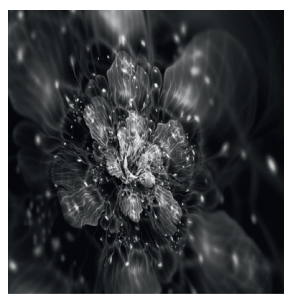

(a)

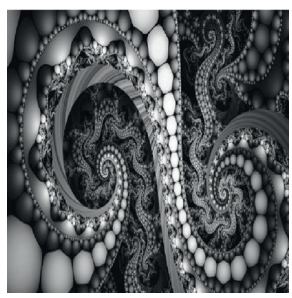

(c)

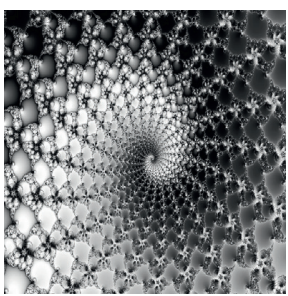

(b)

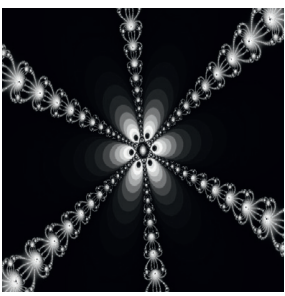

(d)

Figure 9: Graphics to be spliced.

TABLE 1: Example image's gray-level co-occurrence matrix extraction features.

\begin{tabular}{lccc}
\hline Serial number & ASB & ENT & H \\
\hline $\mathrm{a}$ & 0.636 & 0.771 & 3.04 \\
$\mathrm{~b}$ & 0.45 & 1.107 & 2.01 \\
$\mathrm{c}$ & 0.271 & 1.803 & 0.879 \\
$\mathrm{~d}$ & 0.315 & 2.268 & 1.012 \\
\hline
\end{tabular}

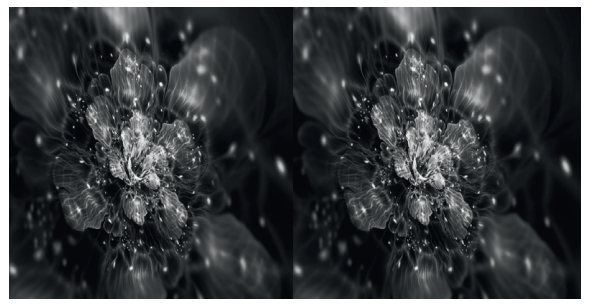

Scattered splicing

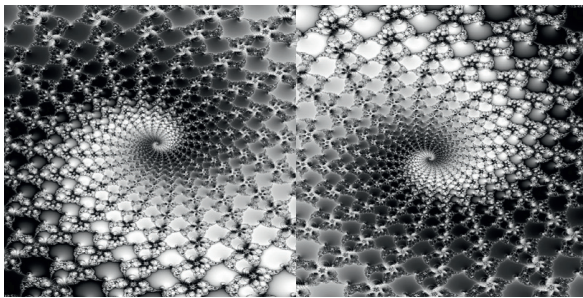

Continuous splicing

Figure 10: Scattered splicing and continuous splicing. 


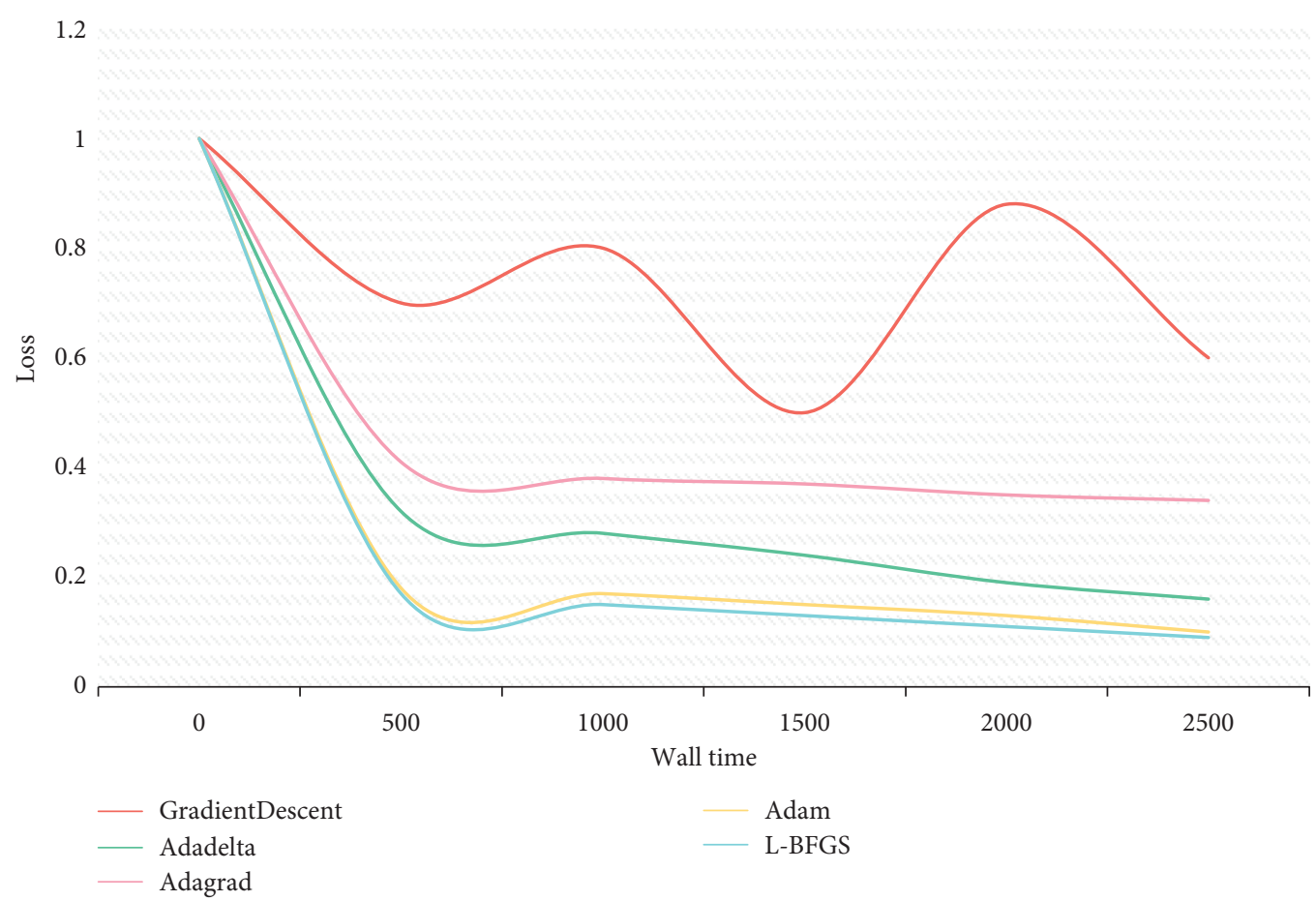

FIgURE 11: Performance comparison of various algorithms.

\section{Conclusion}

Traditional Chinese symbols are the essence of Chinese plain text and are treasures with beautiful meaning and aesthetic value that have been handed down from primitive society. Under the current situation that contemporary smart product modeling is more imitating and learning from the West, designers are more responsible and responsible. The application of traditional Chinese symbols to intelligent products requires profound cultural accumulation from the form of symbols to the appearance of products, from the Confucian or Taoist thoughts represented by symbols to the traditional charm and philosophy of China. Traditional symbols should be refined, and the cultural connotation should have in-depth design. The traditional symbols of Zhongtong should be applied to smart products, and the design of Chinese smart products should be expanded. Market and national characteristics. The disadvantage of this article is that the display of fractal graphics is too complicated and maybe more popular with young people, and it does not cater to all ages. Finally, the artistic value of fractal art graphics is not just the current graphics themselves. I hope that related researchers can further explore the artistic value of graphics and combine technology and art perfectly.

\section{Data Availability}

No data were used to support this study.

\section{Conflicts of Interest}

The author declares that there are no conflicts of interest regarding the publication of this article.

\section{Acknowledgments}

The research was not specifically funded.

\section{References}

[1] N. Krishnaraj, M. Elhoseny, E. L. Lydia, K. Shankar, and O. ALDabbas, "An efficient radix trie-based semantic visual indexing model for large-scale image retrieval in cloud environment," Software: Practice and Experience, vol. 51, 2020.

[2] F. Song, "Inheriting traditional culture and building city memory-taking Qingdao city memory culture center as an Example," International Journal of Social Science and Education Research, vol. 2, no. 5, pp. 57-61, 2019.

[3] Q. Jia, K. Zhao, and H. Yu, "Art design education in the new era featured with the integration of arts and motion sensing technology," Eurasia Journal of Mathematics, Science and Technology Education, vol. 13, no. 8, pp. 5883-5891, 2017.

[4] W. Zhu, "Study of creative thinking in digital media art design education," Creative Education, vol. 11, no. 2, pp. 77-85, 2020.

[5] A. Jeavons, "What is artificial intelligence?" Research World, vol. 2017, no. 65, Article ID 20554, 75 pages, 2017.

[6] D. Hassabis, D. Kumaran, C. Summerfield, and M. Botvinick, "Neuroscience-inspired artificial intelligence," Neuron, vol. 95, no. 2, pp. 245-258, 2017. 
[7] R. Li, Z. Zhao, X. Zhou et al., "Intelligent 5G: when cellular networks meet artificial intelligence," IEEE Wireless Communications, vol. 24, no. 5, pp. 175-183, 2017.

[8] P. Glauner, J. A. Meira, P. Valtchev, R. State, and F. Bettinger, "The challenge of non-technical loss detection using artificial intelligence: a survey," International Journal of Computational Intelligence Systems, vol. 10, no. 1, pp. 760-775, 2017.

[9] M. Hutson, "Artificial intelligence faces reproducibility crisis," Science, vol. 359, no. 6377, pp. 725-726, 2018.

[10] C. Cath, S. Wachter, B. Mittelstadt, M. Taddeo, and L. Floridi, "Artificial intelligence and the "good society": the US, EU, and UK approach," Science and Engineering Ethics, vol. 24, no. 7625, pp. 1-24, 2017.

[11] A. F. Chen, A. C. Zoga, and A. R. Vaccaro, "Point/counterpoint: artificial intelligence in healthcare," Healthcare Transformation, vol. 2, no. 2, pp. 84-92, 2017.

[12] T. Yang, A. A. Asanjan, E. Welles, X. Gao, S. Sorooshian, and $\mathrm{X}$. Liu, "Developing reservoir monthly inflow forecasts using artificial intelligence and climate phenomenon information," Water Resources Research, vol. 53, no. 4, pp. 2786-2812, 2017.

[13] L. Caviglione, M. Gaggero, J. F. Lalande, W. Mazurczyk, and M. Urbański, "Seeing the unseen: revealing mobile malware hidden communications via energy consumption and artificial intelligence," IEEE Transactions on Information Forensics \& Security, vol. 11, no. 4, pp. 799-810, 2017.

[14] M. Nasr, A. E. D. Mahmoud, M. Fawzy, and A. Radwan, "Artificial intelligence modeling of cadmium(II) biosorption using rice straw," Applied Water Science, vol. 7, no. 2, pp. 823-831, 2017.

[15] E. Burton, J. Goldsmith, S. Koenig, B. Kuipers, N. Mattei, and T. Walsh, "Ethical considerations in artificial intelligence courses," AI Magazine, vol. 38, no. 2, pp. 22-34, 2017.

[16] J. Bryson and A. Winfield, "Standardizing ethical design for artificial intelligence and autonomous systems," Computer, vol. 50, no. 5, pp. 116-119, 2017.

[17] S. Price and P. A. Flach, "Computational support for academic peer review: a perspective from artificial intelligence," Communications of the ACM, vol. 60, no. 3, pp. 70-79, 2017.

[18] R. Chatila, K. Firth-Butterflied, J. C. Havens, and K. Karachalios, "The IEEE global initiative for ethical considerations in artificial intelligence and autonomous systems [standards]," IEEE Robotics \& Automation Magazine, vol. 24, no. 1, p. 110, 2017.

[19] Y. U. Bin and K. Kumbier, "Artificial intelligence and statistics," Frontiers of Information Technology \& Electronic Engineering, vol. 19, no. 1, pp. 6-9, 2018.

[20] A. Agrawal, J. S. Gans, and A. Goldfarb, "What to expect from artificial intelligence," MIT Sloan Management Review, vol. 58, no. 3, pp. 23-26, 2017.

[21] D. L. Labovitz, L. Shafner, M. Reyes Gil, D. Virmani, and A. Hanina, "Using artificial intelligence to reduce the risk of nonadherence in patients on anticoagulation therapy," Stroke, vol. 48, no. 5, pp. 1416-1419, 2017.

[22] M. Polina, O. Lucy, Y. Yury et al., "Converging blockchain and next-generation artificial intelligence technologies to decentralize and accelerate biomedical research and healthcare," Oncotarget, vol. 9, no. 5, pp. 5665-5690, 2018.

[23] E.-J. Lee, Y.-H. Kim, N. Kim, and D.-W. Kang, "Deep into the brain: artificial intelligence in stroke imaging," Journal of Stroke, vol. 19, no. 3, pp. 277-285, 2017.

[24] H. Lee, F. M. Troschel, S. Tajmir et al., "Pixel-level deep segmentation: artificial intelligence quantifies muscle on computed tomography for body morphometric analysis," Journal of Digital Imaging, vol. 30, no. 4, pp. 487-498, 2017.
[25] S. Yeung, N. L. Downing, L. Fei-Fei, and A. Milstein, "Bedside computer vision - moving artificial intelligence from driver assistance to patient safety," New England Journal of Medicine, vol. 378, no. 14, pp. 1271-1273, 2018.

[26] M. Taddeo and L. Floridi, "Regulate artificial intelligence to avert cyber arms race," Nature, vol. 556, no. 7701, pp. 296-298, 2018. 